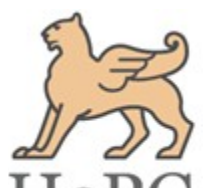

\title{
Influence of nitrogen and phosphorus on the growth and yield of BRRI dhan57
}

\author{
Mahbubul Haque Imrul, ${ }^{1}$ Mst. Afrose Jahan, ${ }^{1}$ Mominul Haque Rabin, ${ }^{2 *}$ Md. Abubakar \\ Siddik, ${ }^{3}$ Shahidul Islam, ${ }^{3}$ Marjana Yeasmin ${ }^{4}$ \\ ${ }^{1}$ Department of Soil Science, Sher-e-Bangla Agricultural University, Sher-e-Bangla Nagar, Dhaka-1207, Bangladesh \\ ${ }^{2}$ Department of Agricultural Chemistry, Sher-e-Bangla Agricultural University, Sher-e-Bangla Nagar, Dhaka-1207, Bangladesh \\ ${ }^{3}$ Department of Agricultural Botany, Sher-e-Bangla Agricultural University, Sher-e-Bangla Nagar, Dhaka-1207, Bangladesh \\ ${ }^{4}$ Department of Agronomy, Sher-e-Bangla Agricultural University, Sher-e-Bangla Nagar, Dhaka-1207, Bangladesh
}

\section{Article history}

Received: 07 April 2016

Accepted: 02 May 2016

Published: 07 May 2016

(C) Imrul et al. (2016)

Publisher

Horizon e-Publishing Group

Corresponding Author

Mominul Haque Rabin

$\square_{\text {robin 94sau@yahoo.com }}$

\begin{abstract}
The field experiment was carried out during the period from July to November, 2013 at the research farm of Sher-e-Bangla Agricultural University, Dhaka, Bangladesh to investigate the influence of nitrogen and phosphorus on the growth and yield of BRRI dhan57. The two factorial experiments were laid out in a Randomized Complete Block Design (RCBD) with three replications. Four levels of nitrogen $\mathrm{N}_{0}: 0 \mathrm{~kg} \mathrm{~N} \mathrm{ha}^{-1}, \mathrm{~N}_{1}: 90 \mathrm{~kg} \mathrm{~N}$

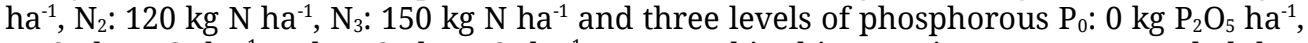
$\mathrm{P}_{1}: 25 \mathrm{~kg} \mathrm{P}_{2} \mathrm{O}_{5}$ ha $^{-1}$ and $\mathrm{P}_{2}: 35 \mathrm{~kg} \mathrm{P}_{2} \mathrm{O}_{5}$ ha $^{-1}$ were used in this experiment. Data revealed that at 30 and 60 days after transplanting (DAT) and at the time of harvest the tallest plants were observed in the treatments $\mathrm{N}_{2}\left(120 \mathrm{~kg} \mathrm{~N} \mathrm{ha}^{-1}\right), \mathrm{P}_{2}\left(35 \mathrm{~kg} \mathrm{P} \mathrm{ha}^{-1}\right)$ and treatment combination $\mathrm{N}_{2} \mathrm{P}_{2}\left(120 \mathrm{~kg} \mathrm{~N}\right.$ and $\left.35 \mathrm{~kg} \mathrm{P} \mathrm{ha}^{-1}\right)$ whereas, the treatments $\mathrm{N}_{0}, \mathrm{P}_{0}$ and treatment combination $\mathrm{N}_{0} \mathrm{P}_{0}$ (without $\mathrm{N}$ and $\mathrm{P}$ ) showed the smallest plant height in each case, respectively. Side by side, $\mathrm{N}_{2}, \mathrm{P}_{2}$ treatments and $\mathrm{N}_{2} \mathrm{P}_{2}$ treatment combination gave the maximum effective tillers hill-1 ${ }^{-1}$, highest length of panicle and the maximum filled grains panicle ${ }^{-1}$. The highest 1000 grain weight $(20.85 \mathrm{~g})$, grain yield $\left(4.95 \mathrm{t} \mathrm{ha}^{-1}\right)$, straw yield $\left(5.39 \mathrm{t} \mathrm{ha}^{-1}\right)$ and biological yield $\left(10.34 \mathrm{t} \mathrm{ha}^{-1}\right)$ were found in the treatment combination $\mathrm{N}_{2} \mathrm{P}_{2}$ and also found highest in each individual under $\mathrm{N}_{2}$ and $\mathrm{P}_{2}$ treatments.
\end{abstract}

\section{Keywords}

Rice; nitrogen; phosphorus; growth; yield

Imrul, M. H., M. A. Jahan, M. H. Rabin, M. A. Siddik, S. Islam, and M. Yeasmin. 2016. Influence of nitrogen and phosphorus on the growth and yield of BRRI dhan57. Plant Science Today 3(2): 175-185. http://dx.doi.org/10.14719/pst.2016.3.2.230

\section{Introduction}

Rice (Oryza sativa L.) is one of the leading cereal crops under Poaceae family. Nearly half of the population of the world use rice as their main food. Maximum Asian people entirely live on rice and above $90 \%$ of total world rice produced and consumed in Asia (BBS, 2013). It has major role in Bangladesh economy providing significant contribution to the Gross Domestic Product (GDP), employment generation and food availability. Rice is widely cultivated cereal crop in Bangladesh. It fulfills about $75 \%$ of the calories and $55 \%$ of the protein of our average daily diet (Bhuiyan et al., 2002). In our climatic and edaphic conditions rice grown well throughout the year. BBS (2013) reported that rice offers about $48 \%$ of rural employment, twothird of total calorie and one-half of the total protein ingestion of an average person in the country. Nearly $75 \%$ of the total cropped area and above $80 \%$ of the total irrigated area is covered by rice. Therefore, it plays a key role in 
Table 1. Records of meteorological observation (monthly) for the period of experiment (June 2013-November 2013)

\begin{tabular}{|c|c|c|c|c|c|}
\hline \multirow[t]{2}{*}{ Year } & \multirow[t]{2}{*}{ Month } & \multicolumn{2}{|c|}{ Air temperature $\left({ }^{\circ} \mathrm{C}\right)$} & \multirow{2}{*}{$\begin{array}{l}\text { Relative humidity } \\
\text { (\%) }\end{array}$} & \multirow{2}{*}{$\begin{array}{c}\text { Total } \\
\text { rainfall (mm) }\end{array}$} \\
\hline & & Maximum & Minimum & & \\
\hline \multirow{6}{*}{2013} & June & 32.8 & 17.4 & 69.4 & 2.70 \\
\hline & July & 31.75 & 16.14 & 71.42 & 2.39 \\
\hline & August & 31.02 & 15.27 & 74.41 & 1.70 \\
\hline & September & 31.46 & 14.82 & 73.20 & 2.10 \\
\hline & October & 30.18 & 14.85 & 67.82 & 1.40 \\
\hline & November & 28.10 & 6.88 & 58.18 & 0.52 \\
\hline
\end{tabular}

the livelihood of the people of Bangladesh. Among the rice growing countries, Bangladesh occupies third position in rice area and fourth position in rice production (BRRI, 2012). But the average yield is relatively low compared to top rice producing countries. The largest part of the total production of rice comes from Aman rice. In Bangladesh about $50.92 \%$ of the rice areas covered by T. aman of which modern T. aman varieties covers $60 \%$ (BBS, 2005). Nitrogen (N) is the essential plant nutrient which is the promising factor in rice production. It is the limiting nutrient in rice production and when applied as inorganic sources then losses highly (Fillery et al. 1984). Effective tillers plant ${ }^{-1}$, yield and yield parameters are significantly influenced by nitrogen on the rice production. (Jashim et al., 1984, BRRI, 1990). So it is needed to find out the appropriate dose of nitrogen fertilizer for effective management and better yield of rice. For normal growth and development, plant required macronutrients like phosphorus (P). The soil chemical properties greatly influence the phosphorus requirements of plant (Sahrawat et al., 2001). The optimum proportion of nitrogen and phosphorus fertilizer enhances the growth and development of a crop as well as ensured the availability of other essential nutrients for the plant. In the light of the above discussion, the present study was undertaken with the following objective: to select the suitable combination of $\mathrm{N}$ and $\mathrm{P}$ fertilizers concerning to growth and yield of BRRI dhan 57 .

\section{Materials and Methods}

\section{Experimental site and climate}

The experiment was carried out in typical rice growing clay loam soil at the farm of Sher-e-Bangla Agricultural University, Dhaka. The experimental area is located in the sub tropical humid condition with medium rainfall during the kharif season (March-September) and scanty rainfall in the rabi season (October-February). During the crop growing period, the mean of minimum and maximum temperature, mean relative humidity and total rainfall have been presented in Table 1.

\section{Experimental treatments}

The experiment was consisted on four levels of nitrogen $\mathrm{N}_{0}: 0 \mathrm{~kg} \mathrm{~N} \mathrm{ha}^{-1}, \mathrm{~N}_{1}: 90 \mathrm{~kg} \mathrm{~N} \mathrm{ha}^{-1}, \mathrm{~N}_{2}: 120 \mathrm{~kg}$
$\mathrm{N} \mathrm{ha}^{-1}, \mathrm{~N}_{3}: 150 \mathrm{~kg} \mathrm{~N} \mathrm{ha}^{-1}$ and three levels of phosphorous $\mathrm{P}_{0}: 0$ kg $\mathrm{P}_{2} \mathrm{O}_{5}$ ha $^{-1}, \mathrm{P}_{1}: 25 \mathrm{~kg} \mathrm{P}_{2} \mathrm{O}_{5}$ ha $^{-1}$ and $\mathrm{P}_{2}: 35 \mathrm{~kg} \mathrm{P}_{2} \mathrm{O}_{5}$ ha $^{-1}$. There were in total $12(4 \times 3)$ treatment combinations such as $\mathrm{N}_{0} \mathrm{P}_{0}, \mathrm{~N}_{0} \mathrm{P}_{1}, \mathrm{~N}_{0} \mathrm{P}_{2}$, $\mathrm{N}_{1} \mathrm{P}_{0}, \mathrm{~N}_{1} \mathrm{P}_{1}, \mathrm{~N}_{1} \mathrm{P}_{2}, \mathrm{~N}_{2} \mathrm{P}_{0}, \mathrm{~N}_{2} \mathrm{P}_{1}, \mathrm{~N}_{2} \mathrm{P}_{2}, \mathrm{~N}_{3} \mathrm{P}_{0}, \mathrm{~N}_{3} \mathrm{P}_{1}$ and $\mathrm{N}_{3} \mathrm{P}_{2}$.

\section{Land preparation}

The land was ploughed by power tiller. Laddering helped breaking the clods and leveling the land followed every ploughing. Before transplanting each unit of plot was cleaned by removing the weeds, stubbles and crop residues. Finally each plot was prepared by puddling for seedling transplanting.

\section{Planting materials, design and plot size}

BRRI dhan57 was used as planting material in this experiment. This variety was developed at the Bangladesh Rice Research Institute from the cross between Bashmoti (D) and BR5 in 1998. It is recommended for Aman season. The two factorial experiments were laid out in a RCBD design with three replications. The size of the each plot was 3.0 $\mathrm{m} \times 1.5 \mathrm{~m}$. The distances between block to block and plot to plot were $1.0 \mathrm{~m}$ and $0.5 \mathrm{~m}$, respectively.

\section{Fertilizer application}

Urea, Triple Super Phosphate (TSP), Muriate of Potash (MOP), Gypsum, zinc sulphate and borax were applied as the source of N, P, K, S, Zn and B respectively. Doses of nitrogen and phosphorus were applied as per treatments. During the final land preparation the one third amount of urea and entire amount of TSP, MOP (120 kg ha-1), gypsum (30 kg ha-1), zinc sulphate (2 $\left.\mathrm{kg} \mathrm{ha}^{-1}\right)$ and borax (10 $\mathrm{kg} \mathrm{ha}^{-1}$ ) were applied. At the tillering and panicle initiation stages, rest of urea was applied in two equal instalments (BRRI, 2013).

\section{Raising of seedlings}

The seedlings of rice were raised wet-bed methods. The seeds were sprouted by soaking for 72 hours. The sprouted seeds were sown uniformly in the well-prepared seed bed. During seedling growing, no fertilizers were used. Proper water and pest 
management practices were followed whenever required.

\section{Transplanting}

The 30 days old nursery seedlings of BRRI dhan57 were uprooted carefully and were kept in soft mud in shade. The seedlings were then transplanted with $20 \mathrm{~cm} \times 20 \mathrm{~cm}$ spacing on the well puddled plots. After one week of transplanting, all plots were checked for any missing hill, which was filled up with extra seedlings whenever required.

\section{Statistical analysis}

All the collected data on different parameters were statistically analyzed following the analysis of variance (ANOVA) technique using MSTAT-C computer package program and mean differences adjusted by Least Significant Difference (LSD) test at $5 \%$ level of significance.

\section{Results and Discussion \\ Plant height}

Plant height of BRRI dhan57 was statistically significant due to different levels of nitrogen at 30 and 60 days after transplanting (DAT) and at harvest (Table 2). At 30 and 60 DAT and harvest, the tallest plant $(22.60,80.81$ and $115.66 \mathrm{~cm}$, respectively) were recorded from $\mathrm{N}_{2}\left(120 \mathrm{~kg} \mathrm{~N} \mathrm{ha}^{-1}\right)$ which were statistically similar $(22.47,80.09$ and $112.44 \mathrm{~cm}$, respectively) with $\mathrm{N}_{3}\left(150 \mathrm{~kg} \mathrm{~N} \mathrm{ha}^{-1}\right)$ whereas the shortest plant $(20.98,61.08$ and 82.09 $\mathrm{cm}$, respectively) was found from $\mathrm{N}_{0}$ or control condition. It was revealed that increased plant height up to a certain level then decreases due to increasing the nitrogen fertilizer. Optimum nitrogen $(\mathrm{N})$ is essential for vegetative growth but excess $\mathrm{N}$ may cause excessive vegetative growth, prolong the growth duration and delay crop maturity with reduction in grain yield. BRRI (1992) reported that 120 to $160 \mathrm{~kg} \mathrm{~N} \mathrm{ha}^{-1}$ was assumed to be due to excessive vegetative growth follower by lodging after flowering. Andrade and Amorim (1996) observed that increasing level of $\mathrm{N}$ increased plant height. Patel and Upadhaya (1993) found that rate of $\mathrm{N}$ up to $150 \mathrm{~kg} \mathrm{ha}^{-1}$ which increased the plant height significantly.

Plant height of BRRI dhan57 varied significantly for different levels of phosphorus at 30 and 60 DAT and harvest (Table 1). At 30 and 60 DAT and harvest, the tallest plant (22.37, 78.12 and $111.64 \mathrm{~cm}$, respectively) were observed from $\mathrm{P}_{2}$ (35 $\mathrm{kg} \mathrm{P}_{2} \mathrm{O}_{5}$ ha $^{-1}$ ) which were statistically identical (22.13, 71.90 and $95.51 \mathrm{~cm}$, respectively) with $\mathrm{P}_{1}$ (25 $\mathrm{kg} \mathrm{P}_{2} \mathrm{O}_{5}$ ha $^{-1}$ ). Whereas the shortest plant (18.85, 59.67 and $80.97 \mathrm{~cm}$, respectively) were observed from $\mathrm{P}_{0}\left(0 \mathrm{~kg} \mathrm{P}_{2} \mathrm{O}_{5} \mathrm{ha}^{-1}\right)$. It revealed that with the increase of application of phosphorus, plant height showed increasing trend, but after a certain level plant height increases very slowly.

Interaction effect of different levels of nitrogen and phosphorus showed significant variation on plant height of BRRI dhan57 at 30 and 60 DAT and harvest (Table 3). At 30 and 60 DAT and harvest, the tallest plant $(28.77,81.27$ and

Table 2. The effect of different levels of nitrogen and phosphorous on plant height of BRRI dhan57

\begin{tabular}{|c|c|c|c|}
\hline \multirow{2}{*}{ Treatment } & \multicolumn{3}{|c|}{ Plant height (cm) } \\
\hline & 30 DAT & $60 \mathrm{DAT}$ & Harvest \\
\hline \multicolumn{4}{|l|}{ Levels of nitrogen } \\
\hline $\mathrm{N}_{0}$ & $20.98 \mathrm{c}$ & $61.08 \mathrm{c}$ & $82.09 \mathrm{c}$ \\
\hline $\mathrm{N}_{1}$ & $21.76 \mathrm{~b}$ & $72.43 \mathrm{~b}$ & $94.31 \mathrm{~b}$ \\
\hline $\mathrm{N}_{2}$ & $22.60 \mathrm{a}$ & $80.81 \mathrm{a}$ & $115.66 \mathrm{a}$ \\
\hline $\mathrm{N}_{3}$ & $22.47 \mathrm{a}$ & $80.09 \mathrm{a}$ & $112.44 \mathrm{a}$ \\
\hline $\operatorname{LSD}_{(0.05)}$ & 0.688 & 0.285 & 5.416 \\
\hline Significance level & 0.01 & 0.01 & 0.01 \\
\hline \multicolumn{4}{|l|}{ Levels of phosphorous } \\
\hline $\mathrm{P}_{0}$ & $18.85 \mathrm{~b}$ & $59.67 \mathrm{c}$ & $80.97 \mathrm{c}$ \\
\hline $\mathrm{P}_{1}$ & $22.13 \mathrm{a}$ & $71.90 \mathrm{ab}$ & $95.51 \mathrm{ab}$ \\
\hline $\mathrm{P}_{2}$ & $22.37 \mathrm{a}$ & $78.12 \mathrm{a}$ & $111.64 \mathrm{a}$ \\
\hline $\operatorname{LSD}_{(0.05)}$ & 0.596 & 0.247 & 4.690 \\
\hline Significance level & 0.01 & 0.01 & 0.01 \\
\hline $\mathrm{CV}(\%)$ & 6.15 & 10.06 & 6.81 \\
\hline
\end{tabular}

$\mathrm{N}_{0}: 0 \mathrm{~kg} \mathrm{~N} \mathrm{ha}^{-1}$ (control); $\mathrm{N}_{1}: 90 \mathrm{~kg} \mathrm{~N} \mathrm{ha}^{-1} ; \mathrm{N}_{2}: 120 \mathrm{~kg} \mathrm{~N} \mathrm{ha}^{-1} ; \mathrm{N}_{3}: 150 \mathrm{~kg} \mathrm{~N} \mathrm{ha}^{-1}$ and $\mathrm{P}_{0}: 0 \mathrm{~kg} \mathrm{P}_{2} \mathrm{O}_{5}$ ha $^{-1}$ (control); $\mathrm{P}_{1}: 25 \mathrm{~kg} \mathrm{P}_{2} \mathrm{O}_{5}$ ha-1 $; \mathrm{P}_{2}: 35$ kg $\mathrm{P}_{2} \mathrm{O}_{5}$ ha $^{-1}$ 
Table 3. Interaction effect of different levels of nitrogen and phosphorous on plant height of BRRI dhan57

\begin{tabular}{|c|c|c|c|}
\hline \multirow[t]{2}{*}{ Treatment } & \multicolumn{3}{|c|}{ Plant height $(\mathrm{cm})$} \\
\hline & 30 DAT & 60 DAT & Harvest \\
\hline $\mathrm{N}_{0} \mathrm{P}_{0}$ & $18.13 \mathrm{f}$ & $60.90 \mathrm{e}$ & $87.75 \mathrm{i}$ \\
\hline $\mathrm{N}_{0} \mathrm{P}_{1}$ & $22.28 \mathrm{e}$ & $65.07 \mathrm{de}$ & $98.16 \mathrm{~h}$ \\
\hline $\mathrm{N}_{0} \mathrm{P}_{2}$ & $22.12 \mathrm{e}$ & $64.77 \mathrm{de}$ & $99.93 \mathrm{~g}$ \\
\hline $\mathrm{N}_{1} \mathrm{P}_{0}$ & $23.43 \mathrm{de}$ & 67.01 cde & $102.29 \mathrm{f}$ \\
\hline $\mathrm{N}_{1} \mathrm{P}_{1}$ & $25.28 \mathrm{bcd}$ & $70.97 \mathrm{bcd}$ & $106.63 \mathrm{~d}$ \\
\hline $\mathrm{N}_{1} \mathrm{P}_{2}$ & $25.62 \mathrm{bc}$ & $71.94 \mathrm{bcd}$ & $104.46 \mathrm{e}$ \\
\hline $\mathrm{N}_{2} \mathrm{P}_{0}$ & $22.26 \mathrm{e}$ & $65.62 \mathrm{de}$ & $96.99 \mathrm{~h}$ \\
\hline $\mathrm{N}_{2} \mathrm{P}_{1}$ & $26.86 \mathrm{~b}$ & $77.21 \mathrm{ab}$ & $114.20 \mathrm{~b}$ \\
\hline $\mathrm{N}_{2} \mathrm{P}_{2}$ & 28.77 a & 81.27 a & $119.05 \mathrm{a}$ \\
\hline $\mathrm{N}_{3} \mathrm{P}_{0}$ & 23.82 cde & $70.32 \mathrm{bcd}$ & $106.83 \mathrm{~d}$ \\
\hline $\mathrm{N}_{3} \mathrm{P}_{1}$ & $26.12 \mathrm{~b}$ & $76.25 \mathrm{ab}$ & $110.18 \mathrm{c}$ \\
\hline $\mathrm{N}_{3} \mathrm{P}_{2}$ & $25.32 \mathrm{bcd}$ & $73.57 \mathrm{bc}$ & $107.53 \mathrm{~d}$ \\
\hline $\operatorname{LSD}_{(0.05)}$ & 1.913 & 6.934 & 1.191 \\
\hline Significance level & 0.05 & 0.05 & 0.01 \\
\hline CV(\%) & 5.87 & 5.98 & 4.65 \\
\hline
\end{tabular}

$\mathrm{N}_{0}: 0$ kg N ha ${ }^{-1}$ (control); $\mathrm{N}_{1}: 90 \mathrm{~kg} \mathrm{~N} \mathrm{ha}^{-1} ; \mathrm{N}_{2}: 120 \mathrm{~kg} \mathrm{~N} \mathrm{ha}^{-1} ; \mathrm{N}_{3}: 150 \mathrm{~kg} \mathrm{~N} \mathrm{ha}^{-1} ; \mathrm{P}_{0}: 0$ kg $\mathrm{P}_{2} \mathrm{O}_{5}$ ha $^{-1}$ (control); $\mathrm{P}_{1}: 25 \mathrm{~kg} \mathrm{P}_{2} \mathrm{O}_{5} \mathrm{ha}^{-1} ; \mathrm{P}_{2}: 35 \mathrm{~kg}$ $\mathrm{P}_{2} \mathrm{O}_{5}$ ha $^{-1}$

$119.05 \mathrm{~cm}$, respectively) were observed from $\mathrm{N}_{2} \mathrm{P}_{2}$ (120 kg N ha-1 and $35 \mathrm{~kg}_{2} \mathrm{O}_{5} \mathrm{ha}^{-1}$ ) whereas, the shortest plant $(18.13,60.90$ and $87.75 \mathrm{~cm}$, respectively) were recorded at 30 and 60 DAT and harvest, respectively from $\mathrm{N}_{0} \mathrm{P}_{0}\left(0 \mathrm{~kg} \mathrm{~N} \mathrm{ha} \mathrm{N}^{-1}\right.$ and 0 $\left.\mathrm{kg} \mathrm{P}_{2} \mathrm{O}_{5} \mathrm{ha}^{-1}\right)$. Amin et al. (2004) conducted an experiment to evaluate the effect of increased plant density and fertilizer dose on yield of rice variety IR-6. They found that increased fertilizer dose of NPK increase plant height.

\section{Number of effective tillers hill $^{-1}$}

Number of effective tillers hill $^{-1}$ was significantly influenced by different nitrogen levels (Table 4). $\mathrm{N}_{2}$ gave the highest (16.60) number of effective tillers hill $^{-1}$ which was statistically similar (16.47) with $\mathrm{N}_{3}$ whereas $\mathrm{N}_{0}$ or control condition gave the lowest (12.98) number of effective tillers hill-1. BINA (1996) stated that different nitrogen levels significantly influenced the number of effective tillers hill $^{-1}$. Chopra and Chopra (2004) reported that nitrogen had significantly effects on yield attributes of effective tillers hill ${ }^{-1}$ with increasing levels of $\mathrm{N}$ up to $120 \mathrm{~kg} \mathrm{~N} \mathrm{ha}^{-1}$ in rice.

Phosphorous fertilizer doses significantly varied of number of effective tillers hill ${ }^{-1}$ of BRRI dhan57 (Table 4). The highest effective tillers hill ${ }^{-1}$ (16.37) was recorded in $\mathrm{P}_{2}$, which was statistically identical (16.13) with $\mathrm{P}_{1}$ whereas the lowest effective tillers hill ${ }^{-1}$ (13.85) was recorded from $\mathrm{P}_{0}$ or control condition. Panhawar et al. (2011) reported that plant growth and development of root, tillering, early flowering and performs other functions like metabolic activities, particularly in protein synthesis is highly influenced by phosphorus.

Significant interaction effect between nitrogen and phosphorus on number of effective tillers hill ${ }^{-1}$ was observed of BRRI dhan57 (Table 5). $\mathrm{N}_{2} \mathrm{P}_{2}$ showed the maximum (17.87) effective tillers hill ${ }^{-1}$ while $\mathrm{N}_{0} \mathrm{P}_{0}$ or control condition showed the minimum (12.27) effective tillers hill ${ }^{-1}$. Chaturvedi (2005) stated that yield is greatly influenced by number of tillers per unit area especially fertile tillers. At harvest tillers $\mathrm{m}^{-2}$ of rice is increased significantly due to application of nitrogen fertilizer. Yosef Tabar (2012) observed that $150 \mathrm{~kg}$ $\mathrm{ha}^{-1}$ nitrogen with $90 \mathrm{~kg} \mathrm{ha}^{-1}$ phosphorus gave maximum (22.25) fertile tillers. Rasheed et al. (2003) reported that the number of effective tillers per hill was increased when NP levels were increased.

\section{Number of ineffective tillers hill ${ }^{-1}$}

Number of ineffective tillers hill $^{-1}$ differed significantly for variation of nitrogen doses (Table 4). The higher number of ineffective tillers hill-1 (3.16) was found from $\mathrm{N}_{3}$ which was statistically 
Table 4. Effect of different levels of nitrogen and phosphorous on number of effective \& ineffective tillers hill ${ }^{-1}$, filled grains panicle ${ }^{-1}$ and length of panicle of BRRI dhan57

\begin{tabular}{cllll}
\hline \multicolumn{1}{c}{ Treatment } & $\begin{array}{c}\text { Number of effective } \\
\text { tillers hill }\end{array}$ & $\begin{array}{c}\text { Length of } \\
\text { panicle }(\mathrm{cm})\end{array}$ & $\begin{array}{c}\text { Number of } \\
\text { ineffective tillers hill }^{-1}\end{array}$ & $\begin{array}{c}\text { Number of filled } \\
\text { grain panicle }^{-1}\end{array}$ \\
\hline Levels of nitrogen & $12.98 \mathrm{c}$ & $20.57 \mathrm{~b}$ & $3.09 \mathrm{a}$ & $77.09 \mathrm{~b}$ \\
\hline $\mathrm{N}_{0}$ & $15.76 \mathrm{~b}$ & $22.11 \mathrm{a}$ & $3.07 \mathrm{a}$ & $89.31 \mathrm{a}$ \\
$\mathrm{N}_{1}$ & $16.60 \mathrm{a}$ & $23.05 \mathrm{a}$ & $2.27 \mathrm{~b}$ & $90.66 \mathrm{a}$ \\
$\mathrm{N}_{2}$ & $16.47 \mathrm{a}$ & $22.68 \mathrm{a}$ & $3.16 \mathrm{a}$ & $88.44 \mathrm{a}$ \\
$\mathrm{N}_{3}$ & 0.688 & 1.133 & 0.285 & 5.416 \\
\hline LSD $_{(0.05)}$ & 0.01 & 0.01 & 0.01 & 0.01 \\
\hline Significance level & $13.85 \mathrm{~b}$ & $19.76 \mathrm{~b}$ & $2.67 \mathrm{~b}$ & $75.97 \mathrm{~b}$ \\
\hline Levels of phosphorous & $16.13 \mathrm{a}$ & $22.84 \mathrm{a}$ & $2.90 \mathrm{ab}$ & $90.51 \mathrm{a}$ \\
\hline $\mathrm{P}_{0}$ & $16.37 \mathrm{a}$ & $23.70 \mathrm{a}$ & $3.12 \mathrm{a}$ & $92.64 \mathrm{a}$ \\
\hline $\mathrm{P}_{1}$ & 0.596 & 0.981 & 0.247 & 4.690 \\
\hline $\mathrm{P}_{2}$ & 0.01 & 0.01 & 0.01 & 0.01 \\
\hline Significance level & 6.15 & 5.49 & 10.06 & 6.81 \\
\hline $\mathrm{CV}_{(0.05)}$ & & & & \\
\hline
\end{tabular}

$\mathrm{N}_{0}: 0 \mathrm{~kg} \mathrm{~N} \mathrm{ha}^{-1}$ (control); $\mathrm{N}_{1}: 90 \mathrm{~kg} \mathrm{~N} \mathrm{ha}^{-1} ; \mathrm{N}_{2}: 120 \mathrm{~kg} \mathrm{~N} \mathrm{ha}^{-1} ; \mathrm{N}_{3}: 150 \mathrm{~kg} \mathrm{~N} \mathrm{ha}^{-1} ; \mathrm{P}_{0}: 0 \mathrm{~kg} \mathrm{P}_{2} \mathrm{O}_{5}$ ha $^{-1}$ (control); $\mathrm{P}_{1}: 25$ kg $\mathrm{P}_{2} \mathrm{O}_{5} \mathrm{ha}^{-1} ; \mathrm{P}_{2}: 35 \mathrm{~kg}$ $\mathrm{P}_{2} \mathrm{O}_{5}$ ha $^{-1}$

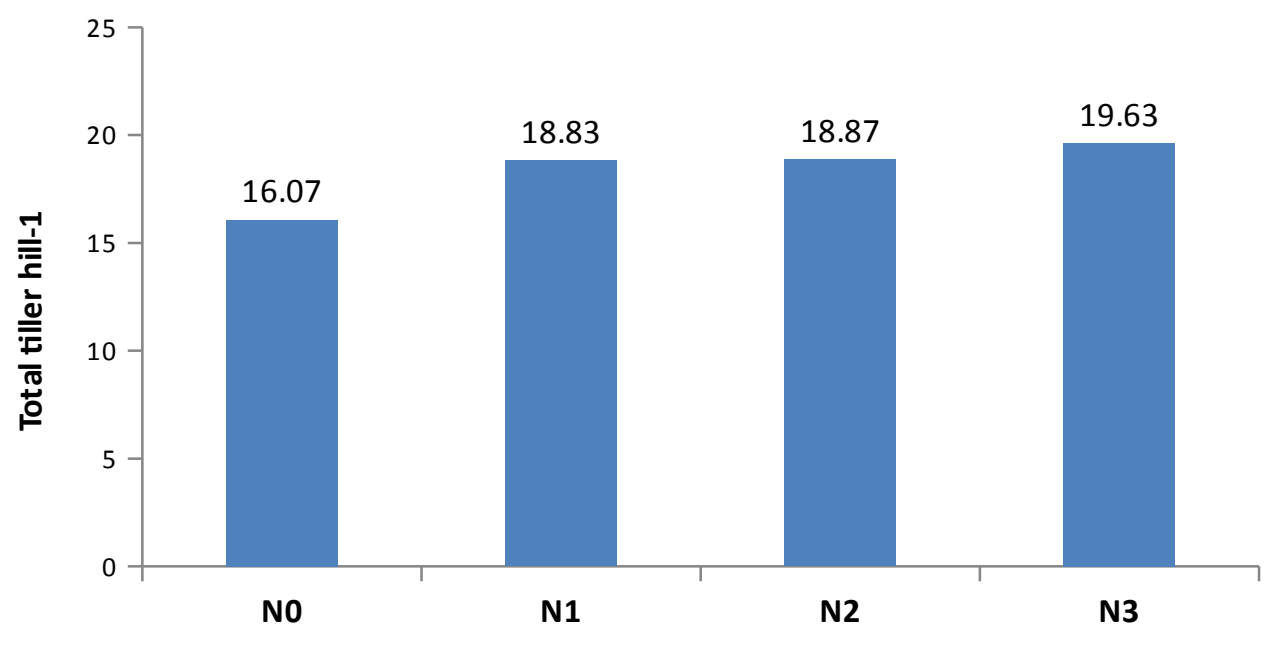

$\mathrm{N}_{0}-0 \mathrm{~kg} \mathrm{ha}^{-1}$ (Control), $\mathrm{N}_{1}-90 \mathrm{~kg} \mathrm{ha}^{-1}, \mathrm{~N}_{2}-120 \mathrm{~kg} \mathrm{ha}^{-1}, \mathrm{~N}_{3}-150 \mathrm{~kg} \mathrm{ha}^{-1}$

Figure 1. Effect of nitrogen on the total tiller number hill-1 of BRRI dhan57

similar (3.09 and 3.07) with $\mathrm{N}_{0}$ and $\mathrm{N}_{1}$, while $\mathrm{N}_{2}$ gave the lower (2.27) number of ineffective tillers hill $^{-1}$.

Number of ineffective tillers hill $^{-1}$ varied significantly for different doses of phosphorous (Table 4). $\mathrm{P}_{2}$ gave the highest (3.12) number of ineffective tillers hill $^{-1}$ which was statistically identical (2.90) with $\mathrm{P}_{1}$. In contrast $\mathrm{P}_{0}$ or control condition gave the lowest (2.67) number of ineffective tillers hill $^{-1}$.

Interaction effect of nitrogen and phosphorus doses showed significant variation on number of ineffective tillers hill $^{-1}$ of BRRI dhan57 (Table 5). The maximum number of ineffective tillers hill ${ }^{-1}$ (3.73) was observed from $\mathrm{N}_{0} \mathrm{P}_{2}$, while the minimum number of ineffective tillers hill $^{-1}$ (2.13) was observed from $\mathrm{N}_{2} \mathrm{P}_{2}$.

\section{Number of total tillers hill $^{-1}$}

Doses of nitrogen had significant effect on number of total tillers hill-1 of BRRI dhan57 (Figure 1). The maximum total tillers hill $^{-1}$ (19.63) was attained from $\mathrm{N}_{3}$ which were statistically similar (18.87 and 18.83) with $\mathrm{N}_{2}$ and $\mathrm{N}_{1}$ and the minimum total 
Table 5. Interaction effect of different doses of nitrogen and phosphorous on number of effective \& ineffective tillers hill $^{-1}$, filled grains panicle ${ }^{-1}$ and length of panicle of BRRI dhan57

\begin{tabular}{|c|c|c|c|c|}
\hline Treatment & $\begin{array}{l}\text { Number of effective } \\
\text { tillers hill }\end{array}$ & $\begin{array}{l}\text { Length of panicle } \\
\qquad(\mathrm{cm})\end{array}$ & $\begin{array}{l}\text { Number of ineffective } \\
\text { tillers hill }^{-1}\end{array}$ & $\begin{array}{l}\text { Number of filled } \\
\text { grain panicle }^{-1}\end{array}$ \\
\hline $\mathrm{N}_{0} \mathrm{P}_{0}$ & $12.27 \mathrm{e}$ & $17.99 \mathrm{e}$ & $2.60 \mathrm{cde}$ & $67.83 \mathrm{e}$ \\
\hline $\mathrm{N}_{0} \mathrm{P}_{1}$ & $13.07 \mathrm{de}$ & $21.49 \mathrm{~cd}$ & $2.93 \mathrm{bc}$ & $76.03 \mathrm{de}$ \\
\hline $\mathrm{N}_{0} \mathrm{P}_{2}$ & $13.60 \mathrm{~cd}$ & $22.22 \mathrm{~cd}$ & $3.73 \mathrm{a}$ & $87.40 \mathrm{bc}$ \\
\hline $\mathrm{N}_{1} \mathrm{P}_{0}$ & $14.47 \mathrm{c}$ & $21.06 \mathrm{~d}$ & $2.93 \mathrm{bc}$ & $80.63 \mathrm{~cd}$ \\
\hline $\mathrm{N}_{1} \mathrm{P}_{1}$ & $16.40 \mathrm{~b}$ & $21.79 \mathrm{~cd}$ & $3.27 \mathrm{ab}$ & $93.13 \mathrm{ab}$ \\
\hline $\mathrm{N}_{1} \mathrm{P}_{2}$ & $16.40 \mathrm{~b}$ & $23.48 \mathrm{bc}$ & $3.00 \mathrm{bc}$ & $94.17 \mathrm{ab}$ \\
\hline $\mathrm{N}_{2} \mathrm{P}_{0}$ & $14.33 \mathrm{~cd}$ & $18.91 \mathrm{e}$ & $2.33 \mathrm{de}$ & $75.00 \mathrm{de}$ \\
\hline $\mathrm{N}_{2} \mathrm{P}_{1}$ & $17.60 \mathrm{ab}$ & $24.64 \mathrm{ab}$ & $2.33 \mathrm{de}$ & 97.23ab \\
\hline $\mathrm{N}_{2} \mathrm{P}_{2}$ & $17.87 \mathrm{a}$ & $25.59 \mathrm{a}$ & $2.13 \mathrm{e}$ & $99.73 \mathrm{a}$ \\
\hline $\mathrm{N}_{3} \mathrm{P}_{0}$ & $14.33 \mathrm{~cd}$ & $21.09 \mathrm{~d}$ & $2.80 \mathrm{bcd}$ & $80.43 \mathrm{~cd}$ \\
\hline $\mathrm{N}_{3} \mathrm{P}_{1}$ & $17.47 \mathrm{ab}$ & $23.45 \mathrm{bc}$ & $3.07 \mathrm{bc}$ & $93.13 \mathrm{ab}$ \\
\hline $\mathrm{N}_{3} \mathrm{P}_{2}$ & $17.60 \mathrm{ab}$ & $23.51 \mathrm{bc}$ & $3.60 \mathrm{a}$ & $91.77 \mathrm{ab}$ \\
\hline $\operatorname{LSD}_{(0.05)}$ & 1.191 & 1.962 & 0.494 & 9.381 \\
\hline Significance level & 0.05 & 0.05 & 0.01 & 0.05 \\
\hline CV(\%) & 6.15 & 5.49 & 10.06 & 6.81 \\
\hline
\end{tabular}

$\mathrm{N}_{0}: 0$ kg N ha ${ }^{-1}$ (control); $\mathrm{N}_{1}: 90 \mathrm{~kg} \mathrm{~N} \mathrm{ha}^{-1} ; \mathrm{N}_{2}: 120 \mathrm{~kg} \mathrm{~N} \mathrm{ha}^{-1} ; \mathrm{N}_{3}: 150 \mathrm{~kg} \mathrm{~N} \mathrm{ha}^{-1} ; \mathrm{P}_{0}: 0$ kg $\mathrm{P}_{2} \mathrm{O}_{5} \mathrm{ha}^{-1}$ (control); $\mathrm{P}_{1}: 25$ kg $\mathrm{P}_{2} \mathrm{O}_{5} \mathrm{ha}^{-1} ; \mathrm{P}_{2}: 35 \mathrm{~kg}$ $\mathrm{P}_{2} \mathrm{O}_{5}$ ha $^{-1}$

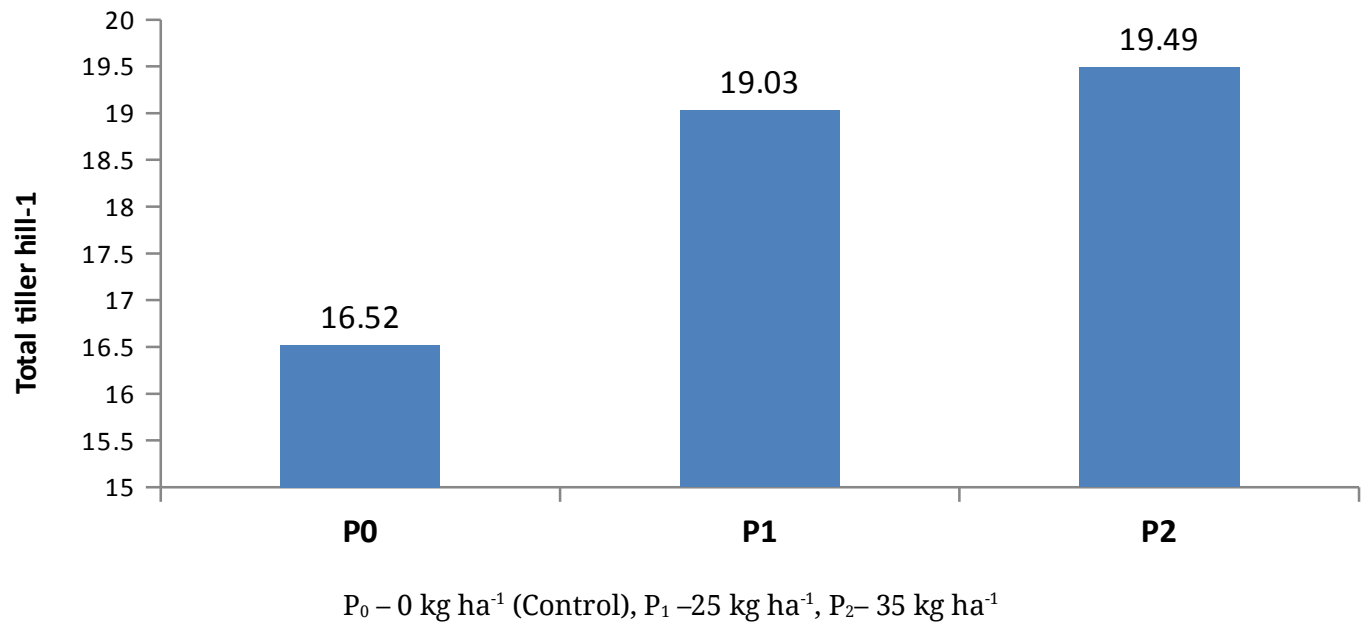

Figure 2. Effect of phosphorus on the total tiller number hill ${ }^{-1}$ of BRRI dhan 57

tillers hill ${ }^{-1}$ (16.07) was observed from $\mathrm{N}_{0}$ or control condition. Bayan and Kandasamy (2002) reported that tillers hill-1 increased with the application of nitrogen fertilizer. Ahmed et al. (2005) found that higher $\mathrm{N}$ dose produced highest effective tiller hill ${ }^{1}$ which ultimately leads to achieve higher total tiller per hill. BINA (1996) also stated that the nitrogen doses had significant effect for tillers number hill-1.

Doses of phosphorus had significant effect on number of total tillers hill-1 ${ }^{-1}$ of BRRI dhan57 (Figure 2). The maximum number of total tillers hill-1 (19.49) was observed from $\mathrm{P}_{2}$, which were statistically identical (19.03) with $\mathrm{P}_{1}$ and the minimum number of total tillers hill $^{-1}$ (16.52) was recorded from $\mathrm{P}_{0}$ or control condition. This result is disagreed with Yosef Tabar (2012) who stated that the phosphorus fertilizer had no significant effect on tiller number. But Alinajoati sisie and Mirshekari (2011) stated that phosphorus is an essential element for tillers development and root growth of wheat.

Interaction effect of nitrogen and phosphorus doses showed significant variation on number of total tillers hill-1 of BRRI dhan57 (Figure 3). The highest total tillers hill-1 $(21.20)$ was recorded from 


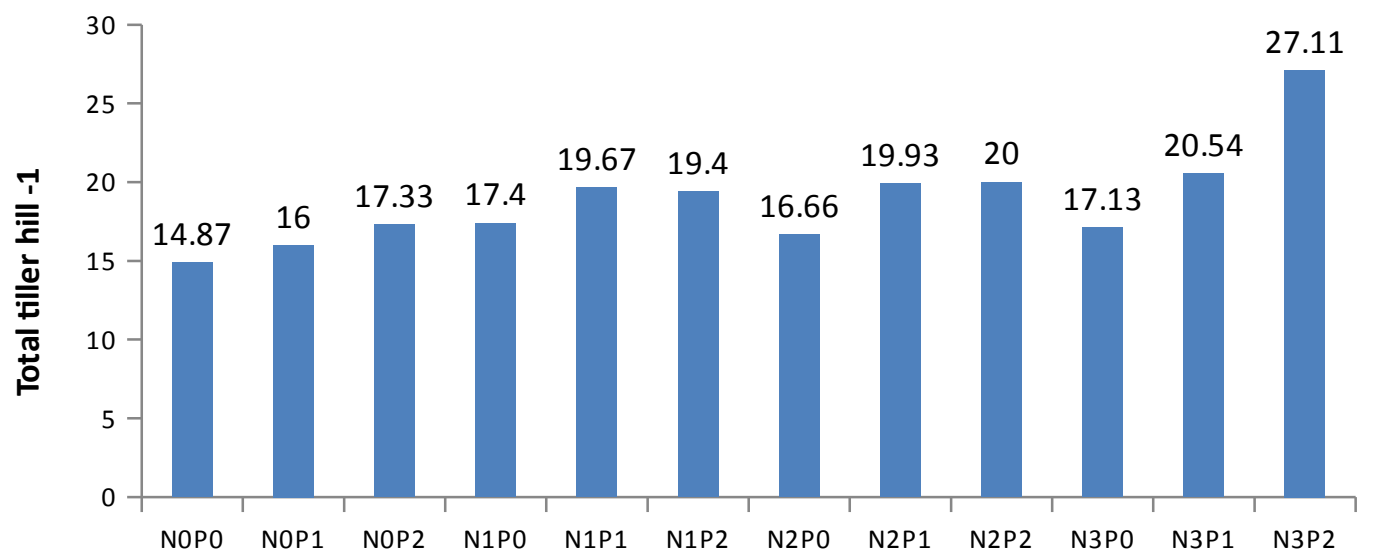

$\mathrm{N}_{0}: 0 \mathrm{~kg} \mathrm{~N} \mathrm{ha}^{-1}$ (control); $\mathrm{N}_{1}: 90 \mathrm{~kg} \mathrm{~N} \mathrm{ha}^{-1} ; \mathrm{N}_{2}: 120 \mathrm{~kg} \mathrm{~N} \mathrm{ha}^{-1} ; \mathrm{N}_{3}: 150 \mathrm{~kg} \mathrm{~N} \mathrm{ha}^{-1} ; \mathrm{P}_{0}: 0$ kg P $\mathrm{P}_{5}$ ha $^{-1}$ (control); $\mathrm{P}_{1}: 25 \mathrm{~kg} \mathrm{P}_{2} \mathrm{O}_{5}$ ha $^{-1} ; \mathrm{P}_{2}: 35 \mathrm{~kg} \mathrm{P}_{2} \mathrm{O}_{5}$ ha $^{-1}$

Figure 3. Effect of phosphorus on the total tiller number hill ${ }^{-1}$ of BRRI dhan 57

Table 6. Effect of different doses of nitrogen and phosphorous on weight of 1000-grains, grain, straw and biological yield of BRRI dhan57

\begin{tabular}{ccccc}
\hline Treatment & $\begin{array}{c}1000 \text { grains weight } \\
\text { (g) }\end{array}$ & $\begin{array}{c}\text { Grain yield } \\
\left(\mathrm{t} \mathrm{ha}^{-1}\right)\end{array}$ & $\begin{array}{c}\text { Straw yield } \\
\left(\mathrm{t} \mathrm{ha}^{-1}\right)\end{array}$ & $\begin{array}{c}\text { Biological yield } \\
\left(\mathrm{t} \mathrm{ha}^{-1}\right)\end{array}$ \\
\hline Levels of nitrogen & & & & \\
\hline $\mathrm{N}_{0}$ & $17.31 \mathrm{~b}$ & $3.15 \mathrm{c}$ & $3.56 \mathrm{c}$ & $6.71 \mathrm{c}$ \\
$\mathrm{N}_{1}$ & $18.68 \mathrm{a}$ & $4.15 \mathrm{~b}$ & $4.57 \mathrm{~b}$ & $8.72 \mathrm{~b}$ \\
$\mathrm{~N}_{2}$ & $19.43 \mathrm{a}$ & $4.56 \mathrm{a}$ & $4.98 \mathrm{a}$ & $9.54 \mathrm{a}$ \\
$\mathrm{N}_{3}$ & $19.33 \mathrm{a}$ & $4.47 \mathrm{a}$ & $4.96 \mathrm{a}$ & $9.43 \mathrm{a}$ \\
\hline LSD $_{(0.05)}$ & 0.989 & 0.243 & 0.235 & 0.421 \\
\hline Significance level & 0.01 & 0.01 & 0.01 & 0.01 \\
\hline Levels of phosphorous & & & & $8.03 \mathrm{~b}$ \\
\hline $\mathrm{P}_{0}$ & $17.50 \mathrm{~b}$ & $3.81 \mathrm{~b}$ & $4.64 \mathrm{~b}$ & $8.82 \mathrm{a}$ \\
$\mathrm{P}_{1}$ & $18.95 \mathrm{a}$ & $4.18 \mathrm{a}$ & $4.69 \mathrm{a}$ & $8.94 \mathrm{a}$ \\
\hline $\mathrm{P}_{2}$ & $19.63 \mathrm{a}$ & $4.25 \mathrm{a}$ & 0.204 & 0.364 \\
\hline LSD & 0.856 & 0.211 & 0.01 & 0.01 \\
\hline Significance level & 0.01 & 0.01 & 5.33 & 5.66 \\
\hline CV(\%) & 6.25 & 8.11 & & \\
\hline
\end{tabular}

$\mathrm{N}_{0}: 0 \mathrm{~kg} \mathrm{~N} \mathrm{ha}^{-1}$ (control); $\mathrm{N}_{1}: 90 \mathrm{~kg} \mathrm{~N} \mathrm{ha}^{-1} ; \mathrm{N}_{2}: 120 \mathrm{~kg} \mathrm{~N} \mathrm{ha}^{-1} ; \mathrm{N}_{3}: 150 \mathrm{~kg} \mathrm{~N} \mathrm{ha}^{-1} ; \mathrm{P}_{0}: 0 \mathrm{~kg} \mathrm{P}_{2} \mathrm{O}_{5}$ ha $^{-1}$ (control); $\mathrm{P}_{1}: 25 \mathrm{~kg} \mathrm{P}_{2} \mathrm{O}_{5}$ ha $^{-1} ; \mathrm{P}_{2}: 35 \mathrm{~kg} \mathrm{P}_{2} \mathrm{O}_{5}$ ha $^{-1}$

$\mathrm{N}_{3} \mathrm{P}_{2}$, while the lowest total tillers hill-1 (14.27) was observed from $\mathrm{N}_{0} \mathrm{P}_{0}$ or control condition. Ndaeyo et al. (2008) conducted an experiment where they found that higher rates of NPK resulted higher number of tillers per plant. Yosef Tabar (2012) reported that the interaction effect of nitrogen and phosphorus fertilizer had significant effect on barrier tiller percentage.

\section{Panicle length}

Panicle length of BRRI dhan57 was statistically influenced by different doses of nitrogen (Table 4). The longest length of panicle $(23.05 \mathrm{~cm})$ was observed from $\mathrm{N}_{2}$ which was statistically similar $(22.11 \mathrm{~cm}$ and $22.68 \mathrm{~cm})$ with $\mathrm{N}_{1}$ and $\mathrm{N}_{3}$, while the shortest length of panicle $(20.57 \mathrm{~cm})$ was found from $\mathrm{N}_{0}$ or control condition. Mondal and Swamy
(2003) reported that application of $\mathrm{N}\left(120 \mathrm{~kg} \mathrm{ha}^{-1}\right)$ as urea in equal splits during transplanting, tillering, panicle initiation and flowering resulted in the highest length of panicle. Ahmed et al. (2005) also found that higher $\mathrm{N}$ dose produced higher panicle length.

Length of panicle of BRRI dhan57 varied significantly for different levels of phosphorous (Table 4). The longest length of panicle $(23.70 \mathrm{~cm})$ was observed from $\mathrm{P}_{2}$ which was statistically identical $(22.84 \mathrm{~cm})$ with $\mathrm{P}_{1}$, whereas the shortest length of panicle $\left(19.76 \mathrm{~cm}\right.$ ) was recorded from $\mathrm{P}_{0}$ or control condition. Das and Sinha (2006) reported that $40 \mathrm{~kg}_{2} \mathrm{O}_{5} \mathrm{ha}^{-1}$ give the better performance in relation to length of panicle of rice.

The panicle length was varied significantly by different doses of nitrogen and phosphorus of 
BRRI dhan57 (Table 5). The longest length of panicle $(25.59 \mathrm{~cm})$ was observed from $\mathrm{N}_{2} \mathrm{P}_{2}$ and the shortest length of panicle $(17.99 \mathrm{~cm})$ from $\mathrm{N}_{0} \mathrm{P}_{0}$ or control condition. Mondal and Swamy (2003) found that application of $\mathrm{N}\left(120 \mathrm{~kg} \mathrm{ha}^{-1}\right)$ as urea in equal splits during transplanting, tillering, panicle initiation and flowering resulted in the highest length of panicle. Duhan and Singh (2002) reported that $40 \mathrm{~kg} \mathrm{P}_{2} \mathrm{O}_{5} \mathrm{ha}^{-1}$ give the better performance in relation to length of panicle of rice.

\section{Number of filled grains panicle ${ }^{-1}$}

Filled grains panicle- ${ }^{1}$ was significantly influenced by doses of nitrogen (Table 4). $\mathrm{N}_{2}$ gave the maximum (90.66) number of filled grains panicle ${ }^{-1}$ which was statistically similar (89.31 and 88.44) with $\mathrm{N}_{1}$ and $\mathrm{N}_{3}$. Whereas the minimum filled grains panicle $^{-1}$ (77.09) was observed in $\mathrm{N}_{0}$ or control condition. Filled grains panicle ${ }^{-1}$ is one of the most important yields contributing parameter in case of grain crops. Bhuyan et al. (2012) observed that bed planting with foliar nitrogen fertilizer produced higher number of grains panicle $^{-1}$. Rahman et al. (2007) conducted an experiment where the results showed that nitrogen level significantly influenced growth and yield components and maximum grains panicle ${ }^{-1}$ was found from $80 \mathrm{~kg} \mathrm{~N} / \mathrm{ha}$.

Number of filled grains panicle ${ }^{-1}$ of BRRI dhan57 varied significantly for different doses of phosphorous (Table 4). The maximum number of filled grains panicle ${ }^{-1}$ (92.64) was observed from $\mathrm{P}_{2}$, which were statistically identical (90.51) with $\mathrm{P}_{1}$ whereas the minimum number of filled grains panicle $^{-1}$ (75.97) was observed from $\mathrm{P}_{0}$ or control condition. Yosef Tabar (2012) stated that the highest total grains panicle ${ }^{-1}$ was recorded for 150 $\mathrm{kg} / \mathrm{ha}$ phosphorus fertilizer. Yoefi et al. (2011) also reported that number of grains panicle ${ }^{-1}$ was significantly influenced by phosphorus fertilizer.

Number of filled grains panicle ${ }^{-1}$ was significantly varied by doses of nitrogen and phosphorus (Table 5). $\mathrm{N}_{2} \mathrm{P}_{2}$ showed the maximum (99.73) filled grains panicle ${ }^{-1}$ whereas $\mathrm{N}_{0} \mathrm{P}_{0}$ or control gave the minimum (67.83) filled grains panicle $^{-1}$. Filed grains panicle ${ }^{-1}$ was most important yield contributing parameter in which was affected the yield of rice (Fallah, 2012). Yosef Tabar (2012) found that maximum fertile spikelet was observed for $150 \mathrm{~kg} \mathrm{ha}^{-1}$ nitrogen with $60 \mathrm{~kg} \mathrm{ha}^{-1}$ phosphorus fertilizer.

\section{0 grains weight}

1000 grains weight showed significant variation due to different doses of nitrogen (Table 6). $\mathrm{N}_{2}$ gave the maximum (19.43 g) 1000 grains weight which was statistically similar (19.33 g and $18.68 \mathrm{~g}$ ) with $\mathrm{N}_{3}$ and $\mathrm{N}_{1}$. While $\mathrm{N}_{0}$ or control condition gave the minimum (17.31 g) weight of 1000-grains. This result agreed with Mondal and Swamy (2003) who reported that $120 \mathrm{~kg} \mathrm{~N} \mathrm{ha}^{-1}$ applied as urea in equal splits during transplanting, tillering, panicle initiation and flowering resulted in the highest 1000-grain weight.

1000 grains weight of BRRI dhan57 varied significantly for doses of phosphorous (Table 6). The maximum 1000 grains weight (19.63 g) was recorded from $\mathrm{P}_{2}$, which were statistically identical (18.95 g) with $\mathrm{P}_{1}$, while the minimum 1000 grains weight $(17.50 \mathrm{~g})$ was found from $\mathrm{P}_{0}$ or control condition. Yosef Tabar (2012) stated that $90 \mathrm{~kg} \mathrm{ha}^{-1}$ phosphorus fertilizer gave the maximum 1000 grains weight.

1000 grains weight was significantly influenced by different doses of nitrogen and phosphorus (Table 7). $\mathrm{N}_{2} \mathrm{P}_{2}$ combination performed better 1000 grains weight $(20.85 \mathrm{~g})$ and $\mathrm{N}_{0} \mathrm{P}_{0}$ or control condition gave the lowest (16.75 g) 1000 grains weight. Yosef Tabar (2012) found that the highest 1000 grains weight with $150 \mathrm{~kg} \mathrm{ha}^{-1}$ nitrogen with $90 \mathrm{~kg} \mathrm{ha}^{-1}$.

\section{Grain yield}

Statistically significant variation was recorded for grain yield ha- ${ }^{1}$ of BRRI dhan57 due to different levels of nitrogen (Table 6). $\mathrm{N}_{2}$ gave the maximum grain yield (4.56 t ha-1) which was statistically similar (4.47 t ha-1) with $\mathrm{N}_{3}$ and closely followed (4.15 $\mathrm{t} \mathrm{ha}^{-1}$ ) by $\mathrm{N}_{1}$, whereas $\mathrm{N}_{0}$ gave the minimum (3.15 t ha ${ }^{-1}$ ) grain yield. Dwibvedi (1997) noticed that grain yield, straw yield as well as harvest index with $60 \mathrm{~kg} \mathrm{~N} \mathrm{ha}^{-1}$ was influenced by different doses of nitrogen. Adhikary and Rhaman (1996) found that the maximum yield was obtained from $100 \mathrm{~kg} \mathrm{~N} \mathrm{ha}^{-1}\left(4.52 \mathrm{t} \mathrm{ha}^{-1}\right)$ and followed by $120 \mathrm{~kg} \mathrm{~N}$ $\mathrm{ha}^{-1}$ (4.46 t ha-1) and $80 \mathrm{~kg} \mathrm{~N} \mathrm{ha}^{-1}$ (4.40 t ha-1).

Grain yield ha $^{-1}$ of BRRI dhan57 varied significantly for different levels of phosphorous (Table 6). The highest grain yield (4.25 $\left.\mathrm{t} \mathrm{ha}^{-1}\right)$ was recorded from $\mathrm{P}_{2}$, which was statistically similar (4.18 t ha-1) with $\mathrm{P}_{1}$ and the lowest grain yield (3.81 $\left.\mathrm{t} \mathrm{ha}^{-1}\right)$ was observed from $\mathrm{P}_{0}$ or control condition. Islam et al. (2010a) reported that grain yield was significantly increased by $10 \mathrm{~kg} \mathrm{ha}^{-1} \mathrm{P}$ but it is not differ by the application of 20 and $30 \mathrm{~kg} \mathrm{P} \mathrm{ha}^{-1}$. Grain yield was significantly affected by phosphorus fertilizer (Yosefi et al., 2011). Panhawar et al. (2011) stated that increase upland rice yield by applying of phosphorus fertilizer. Li et al. (2010) reported that $P$ fertilizer application is one of the most essential for crop yield.

Grain yield ha $^{-1}$ of BRRI dhan57 varied significantly for different levels of nitrogen and phosphorous (Table 7). The maximum grain yield (4.95 $\mathrm{t} \mathrm{ha}^{-1}$ ) was observed from $\mathrm{N}_{2} \mathrm{P}_{2}$, whereas the minimum grain yield $\left(3.07 \mathrm{t} \mathrm{ha}^{-1}\right)$ was recorded from $\mathrm{N}_{0} \mathrm{P}_{0}$ under 'control conditions'. Islam et al. (2008) reported that grain yield influenced significantly due to different rates of nutrients application and 60-19-36 kg/ha NPK maximized the yield of T. aman rice. 
Table 7. Interaction effect of different doses of nitrogen and phosphorous on weight of 1000-grains, grain, straw and biological yield of BRRI dhan57

\begin{tabular}{|c|c|c|c|c|}
\hline Treatment & $\begin{array}{l}1000 \text { grains weight } \\
\text { (g) }\end{array}$ & $\begin{array}{l}\text { Grain yield } \\
\left(\mathrm{t} \mathrm{ha}^{-1}\right)\end{array}$ & $\begin{array}{l}\text { Straw yield } \\
\left(\mathrm{t} \mathrm{ha}^{-1}\right)\end{array}$ & $\begin{array}{l}\text { Biological yield } \\
\left(\mathrm{t} \mathrm{ha}^{-1}\right)\end{array}$ \\
\hline $\mathrm{N}_{0} \mathrm{P}_{0}$ & $16.75 \mathrm{e}$ & $3.07 \mathrm{e}$ & $3.43 \mathrm{e}$ & $6.50 \mathrm{f}$ \\
\hline $\mathrm{N}_{0} \mathrm{P}_{1}$ & $17.15 \mathrm{e}$ & $3.14 \mathrm{e}$ & $3.63 \mathrm{e}$ & $6.76 \mathrm{f}$ \\
\hline $\mathrm{N}_{0} \mathrm{P}_{2}$ & 18.07 cde & $3.23 \mathrm{e}$ & $3.63 \mathrm{e}$ & $6.86 \mathrm{f}$ \\
\hline $\mathrm{N}_{1} \mathrm{P}_{0}$ & $17.95 \mathrm{de}$ & $3.96 \mathrm{~cd}$ & $4.36 \mathrm{~cd}$ & $8.31 \mathrm{de}$ \\
\hline $\mathrm{N}_{1} \mathrm{P}_{1}$ & 18.43 b-e & $4.21 \mathrm{bcd}$ & $4.61 \mathrm{bcd}$ & 8.82 cde \\
\hline $\mathrm{N}_{1} \mathrm{P}_{2}$ & $19.65 \mathrm{a}-\mathrm{d}$ & $4.28 \mathrm{bcd}$ & $4.74 \mathrm{bc}$ & $9.03 \mathrm{~cd}$ \\
\hline $\mathrm{N}_{2} \mathrm{P}_{0}$ & $17.13 \mathrm{e}$ & $3.86 \mathrm{~d}$ & $4.22 \mathrm{~d}$ & $8.08 \mathrm{e}$ \\
\hline $\mathrm{N}_{2} \mathrm{P}_{1}$ & $20.32 \mathrm{ab}$ & $4.87 \mathrm{a}$ & $5.33 \mathrm{a}$ & $10.20 \mathrm{ab}$ \\
\hline $\mathrm{N}_{2} \mathrm{P}_{2}$ & $20.85 \mathrm{a}$ & $4.95 \mathrm{a}$ & $5.39 \mathrm{a}$ & $10.34 \mathrm{a}$ \\
\hline $\mathrm{N}_{3} \mathrm{P}_{0}$ & 18.15 cde & $4.37 \mathrm{bc}$ & $4.87 \mathrm{~b}$ & $9.24 \mathrm{c}$ \\
\hline $\mathrm{N}_{3} \mathrm{P}_{1}$ & $19.88 \mathrm{abc}$ & $4.51 \mathrm{ab}$ & $5.00 \mathrm{ab}$ & $9.52 \mathrm{bc}$ \\
\hline $\mathrm{N}_{3} \mathrm{P}_{2}$ & $19.95 \mathrm{abc}$ & $4.52 \mathrm{ab}$ & $5.01 \mathrm{ab}$ & $9.53 \mathrm{bc}$ \\
\hline $\operatorname{LSD}_{(0.05)}$ & 1.713 & 0.422 & 0.408 & 0.728 \\
\hline Significance level & 0.05 & 0.05 & 0.01 & 0.01 \\
\hline CV(\%) & 6.25 & 8.11 & 5.33 & 5.66 \\
\hline
\end{tabular}

$\mathrm{N}_{0}: 0 \mathrm{~kg} \mathrm{~N} \mathrm{ha}^{-1}$ (control); $\mathrm{N}_{1}: 90 \mathrm{~kg} \mathrm{~N} \mathrm{ha}^{-1} ; \mathrm{N}_{2}: 120 \mathrm{~kg} \mathrm{~N} \mathrm{ha}^{-1} ; \mathrm{N}_{3}: 150 \mathrm{~kg} \mathrm{~N} \mathrm{ha}^{-1} ; \mathrm{P}_{0}: 0 \mathrm{~kg} \mathrm{P}_{2} \mathrm{O}_{5}$ ha $^{-1}$ (control); $\mathrm{P}_{1}: 25 \mathrm{~kg} \mathrm{P}_{2} \mathrm{O}_{5} \mathrm{ha}^{-1} ; \mathrm{P}_{2}: 35 \mathrm{~kg}$ $\mathrm{P}_{2} \mathrm{O}_{5} \mathrm{ha}^{-1}$

\section{Straw yield}

Statistically significant variation was recorded for straw yield ha- ${ }^{1}$ of BRRI dhan57 due to different doses of nitrogen (Table 6). The highest straw yield (4.98 $\mathrm{t} \mathrm{ha}^{-1}$ ) was observed from $\mathrm{N}_{2}$ which was statistically similar (4.96 t ha-1) to $\mathrm{N}_{3}$ and closely followed (4.57 t ha-1) by $\mathrm{N}_{1}$, whereas the lowest straw yield (3.56 t ha-1) was recorded from $\mathrm{N}_{0}$ or control. Mondal and Swamy (2003) reported that $\mathrm{N}$ $\left(120 \mathrm{~kg} \mathrm{ha}^{-1}\right)$ applied as urea in equal splits during transplanting, tillering, panicle initiation and flowering resulted in the highest straw yield. Islam et al. (2008b) found that straw yield significantly influenced by nitrogen levels. This result are in an agreement with the findings of Dhane et al. (1989) who reported that straw yield increases with increasing nitrogen level.

Straw yield ha $^{-1}$ of BRRI dhan57 varied significantly for different levels of phosphorous (Table 6). $\mathrm{P}_{2}$ gave the (4.69 $\left.\mathrm{t} \mathrm{ha}^{-1}\right)$ highest straw yield which was statistically similar $\left(4.64 \mathrm{t} \mathrm{ha}^{-1}\right)$ with $\mathrm{P}_{1}$ whereas $\mathrm{P}_{0}$ or control gave the lowest (4.22 t ha ${ }^{-1}$ ) straw yield. Gebrekidan and Seyoum (2006) reported that harvest index of rice was significantly increased by the application of $13.2 \mathrm{~kg}$ $\mathrm{P} \mathrm{ha}^{-1}$, accordingly harvest index of the rice crop was negatively and significantly correlated with straw yield.
Statistically significant variation was recorded for straw yield ha-1 of BRRI dhan 57 due to different doses of nitrogen and phosphorous (Table 7). The maximum straw yield (5.39 $\left.\mathrm{t} \mathrm{ha}^{-1}\right)$ was obtained from $\mathrm{N}_{2} \mathrm{P}_{2}$, whereas the minimum straw yield $\left(3.43 \mathrm{t} \mathrm{ha}^{-1}\right)$ was recorded from $\mathrm{N}_{0} \mathrm{P}_{0}$ or control. Yosef Tabar (2013) stated that $150 \mathrm{~kg} \mathrm{~N} \mathrm{ha}^{-1}$ with $90 \mathrm{~kg} \mathrm{P} \mathrm{ha}^{-1}$ gave the maximum straw yield.

\section{Biological yield}

Statistically significant variation was recorded for biological yield ha $^{-1}$ of BRRI dhan57 due to different doses of nitrogen (Table 6). $\mathrm{N}_{2}$ gave the highest biological yield $\left(9.54 \mathrm{t} \mathrm{ha}^{-1}\right)$ which was statistically similar $\left(9.43 \mathrm{t} \mathrm{ha}^{-1}\right)$ to $\mathrm{N}_{3}$ and closely followed $\left(8.72 \mathrm{t} \mathrm{ha}^{-1}\right)$ by $\mathrm{N}_{1}$ while $\mathrm{N}_{0}$ gave the lowest $\left(6.71 \mathrm{t} \mathrm{ha}^{-1}\right)$ biological yield. Vegetative growth was influenced due to the higher dose of urea and for the reason the grain and straw yield was also increased with the increased dose of nitrogenous fertilizer. Singh and Lallu (2005) stated that each increment dose of $\mathrm{N}$ significantly increased grain and straw yields (biological yield) over its preceding dose.

Biological yield ha ${ }^{-1}$ of BRRI dhan57 varied significantly for different levels of phosphorous (Table 6). The highest biological yield (8.94 t ha-1) 
was recorded from $\mathrm{P}_{2}$, which were statistically identical (8.82 $\left.\mathrm{t} \mathrm{ha}^{-1}\right)$ with $\mathrm{P}_{1}$, while the lowest biological yield $\left(8.03 \mathrm{t} \mathrm{ha}^{-1}\right)$ was found from $\mathrm{P}_{0}$ or control. Uddin et al. (2014) found that maximum biological yield of BRRI dhan57 obtained from 40 $\mathrm{kg} \mathrm{P} \mathrm{ha-1}$. Yosef Tabar (2013) also stated that $90 \mathrm{~kg} \mathrm{P}$ $\mathrm{ha}^{-1}$ gave the highest biological yield.

Biological yield ha-1 of BRRI dhan57 varied significantly for different levels of nitrogen and phosphorous (Table 7). The highest biological yield (10.34 tha $\mathrm{ha}^{-1}$ ) was observed from $\mathrm{N}_{2} \mathrm{P}_{2}$, whereas the lowest biological yield $\left(6.50 \mathrm{t} \mathrm{ha}^{-1}\right)$ from $\mathrm{N}_{0} \mathrm{P}_{0}$ or control condition. Yosef Tabar (2013) reported that $150 \mathrm{~kg} \mathrm{~N} \mathrm{ha}^{-1}$ with $90 \mathrm{~kg} \mathrm{P} \mathrm{ha}^{-1}$ gave the maximum biological yield.

\section{Conclusion}

Different doses of nitrogen and phosphorus varied significantly for growth and yield of BRRI dhan57. Maximum of yield contributing parameter were in application $120 \mathrm{~kg} \mathrm{ha}^{-1}$ nitrogen fertilizer, also with application $35 \mathrm{~kg} \mathrm{ha}^{-1}$ phosphorus fertilizer. It was revealed that application of $120 \mathrm{~kg} \mathrm{~N} \mathrm{ha}^{-1}$ along with $35 \mathrm{~kg} \mathrm{P}_{2} \mathrm{O}_{5} \mathrm{ha}^{-1}$ has more potential in regarding to yield and yield contributing characters of BRRI dhan57.

\section{Acknowledgements}

Sincere thanks to Sher-e-Bangla Agricultural University, Bangladesh for providing facilities for conducting research.

\section{References}

Adhikary, R. C., and H. Rahman. 1996. Effect of different doses of nitrogen on the yield of BR 11 variety of rice. In: Variety, Fertilizer and Weedicide Trial of some Field crops. Rural Development Academy, Bogra, 15-20.

Ahmed, M., M. Islam, and S. K. Paul, 2005. Effect of nitrogen on yield and other plant characters of Local T. Aman Rice, Var. Jatai. Res J Agric Biol Sci 1(2): 158-161.

Ahsan, K. 1996. Effect of rate of $\mathrm{N}$ fertilizer on growth and yield of japonica and japonica indica hybrid of rice. Bangladesh Rice J 7(1\&2): 17-21.

Alinajati Sisie, S. and B. Mirshekari, 2011. Effect of phosphorus fertilization and seed bio fertilization on harvest index and phoshorus use efficiency of wheat cultivars. Journal of Food, Agriculutral \& Environment 19(2): 388-397.

Amin, M., M. A. Khan, E. A. Khan, and M. Ramzan. 2004. Effect of increased plant density and fertilizer dose on the yield of rice variety Ir-6. J Res Sci 15(1): 0916.

Bayan, H. C. and O. S. Kandasamy, 2002. Effect of weed control methods application of nitrogen on weed and crop in direct seeded puddle rice. Res Hisar 24 (2): 200-272.

BBS (Bangladesh Bureau of Statistics). 2005. The statistical Yearbook of Bangladesh. Bangladesh Bureau of Statistics, Statistics Division, Ministry of
Planning, Govt. of the People's Republic of Bangladesh.

BBS (Bangladesh Bureau of Statistics). 2013. Statistical Yearbook of Bangladesh Statistics Division, Ministry of Planning, Govt. of the People's Republic of Bangladesh, pp. 189-258.

Bhuiya, M. S. U., S. M. A. Hossain, and S. K. G. Kabir. 2002. Nitrogen fertilization in rice cv. BR10 after green manuring. Bangladesh J Agril Sci 16(1): 89-92.

Bhuyan, M. H. M., Mst. R. Ferdousi, and M. T. Iqbal, 2012. Foliar spray of nitrogen fertilizer on raised bed increases yield of transplanted aman rice over conventional method. International Scholarly Research Network, ID 184953.

BINA (Bangladesh Institute of Nuclear Agriculture). 1996. Annul Report for 1993-94. BINA. P.O. Box No. 4, Mymensingh: 175.

BRRI (Bangladesh Rice Research Institute). 1990. Nitrogen response of promising variety. Annual Rep. Bangladesh Rice Res. Inst., Joydebpur, Gazipur, pp: 95.

BRRI (Bangladesh Rice Research Institute). 2012. Annual Report for 2011. Bangladesh Rice Res. Inst. Joydebpur, Gazipur, Bangladesh, pp. 9-13.

BRRI (Bangladesh Rice Research Institute). 2013. Adhunik Dhaner Chash. Joydebpur, Dhaka. p. 19.

Chaturvedi, I. 2005. Effect of nitrogen fertilizers on growth, yield and quality of hybrid rice (oryza sativa L.). Journal of Central European Agriculture 6(4): 611-618.

Chopra, N. K. and N. Chopra, 2004. Seed yield and quality of "Pusa 44" rice (Oryza sativa) as influenced by nitrogen fertilizer and raw spacing. Indian J Agril Sci 74(3): 144-146.

Dhane, S. S., R. R. Khadse, V. H. Patil, and N. K. Sauant, 1989. Effect of deep placed USG with limited green manure on transplanted rice yield. Intl Rice Res Newsl 14(4): 31.

Duhan, B. S. and M. Singh. 2002. Effect of green manuring and nitrogen on the yield and nutrient uptake of micronutrient by rice. J Indian Soc Soil Sci 50(2): 178-180.

Dwivedi, D. K. 1997. Response of scanted rice (Oryza sativa) genotypes to nitrogen under mid-land situation. Indian J Agron 42 (1): 74-76.

Fallah, A. 2012. Interactive effects of nitrogen and irrigation methods on the growth and yield of rice in amol area. Int J Agri 3(4):111-113.

Fillery, I. R. P., J. R. Simpson, and S. K. De Datta. 1984. Influence of field environment and fertilizer management on ammonia loss from flooded rice. $J$ Soil Sci Soc Amer 48: 914-920. doi: 10.2136/sssaj1984.03615995004800040043x

Gebrekidan, H. and M. Seyoum, 2006. Effect of mineral $\mathrm{N}$ and $\mathrm{P}$ fertilizers on yield and yield components of flooded lowland rice on vertisols of fogera plain, Ethiopia. J Agri Rural Devel Trop Subtrop 107(2):161-176.

Gomez, K. A. and A. A. Gomez, 1984. Statistical procedures for Agricultural Research. Jhon Wiley and Sons, New York.

Islam, M. R., P. K. Saha, M. J. Zaman, and S. K. Uddin. 2010. Phosphorus fertilization in inbred and hybrid rice. J Biol Sci 19(2): 181-187. 
Islam, M. S., M. A. Hossain, M. A. H. Chowdhury, and M. A. Hannan, 2008b. Effect of nitrogen and transplanting date on yield and yield components of aromatic rice. $J$ Bangladesh Agril Univ 6(2):291-296.

Islam, M. S., M. I. A. Howlader, S. Rafiquzzaman, H. M. K. Bashar, and M. H. Al-Mamun, 2008a. Yield response of chili and T. aman rice to NPK fertilizers in ganges tidal floodplain. J Soil Nature 2(1): 07-13.

Jashim, C., U. Ahmed, and K. U. Ahmed. 1984. Response of rice varieties to applied $\mathrm{N}$ in saline soils. Int Rice Res Newsl 9(5): 22.

Li, J., J. Gao, Y. Ma, 2010. Phosphorus accumulation in soil in rice-rice cropping systems with chemical fertilizer application: modeling and validation. World congress of soil science, soil solutions for a changing world. 224227.

M. H. Uddin, K. M. Rahman, M. Z. Rahman, Z. Alam, and M. A. Salam, 2014. Effect of phosphorus and sulphur on yield of BRRI dhan57. J Environ Sci \& Natural Resources 7(1): 7-11

Mondal, S. S. and S. N. Swamy, 2003. Effect of time of $\mathrm{N}$ application on yield and yield attributes of rice (Oryza sativa) cultivars. Env Ecol 21(2): 411-413.

Ndaeyo, N. U., K. U. Iboko, G. I. Harry, and S. O. Edem, 2008. Growth and yield performances of some upland rice (oryza sativa l.) Cultivars as influenced by varied rates of NPK (15:15:15) fertilizer on an ultisol. J Trop Agric Food Env Ext 7(3): 249 - 255.

Page, A. L., R. H. Miller, and D. R. Keeney, 1982. Methods of analysis part 2, Chemical and Microbiological Properties, Second Edition American Society of Agronomy, Inc., Soil Science Society of American Inc. Madson, Wisconsin, USA. pp. 403-430.

Panhawar, Q. A. and R. Othman, 2011. Effect of phosphatic fertilizer on root colonization of aerobic rice by phosphate-solubilizing bacteria. International conference on food engineering and biotechnology. IPCBEE (9):145-149.
Patel, P. K. and P. N. Upadhyay, 1993. Response of wheat (Triticum aestivum) to irrigation under varying levels of nitrogen and phosphorus. Indian J Agron 38(1): 113-115.

Rahman, M. H., M. M. Khatun, M. A. A. Mamun, M. Z. Islam, and M. R. Islam, 2007. Effect of number of seedling hill ${ }^{-1}$ and nitrogen level on growth and yield of BRRI dhan32. J Soil Nature 1(2):0107.

Rasheed, M., M. Asif, and A. Ghafoor, 2003. Yield and yield attributes of fine rice BAS-385 as affected by different NP levels on farmer's field. Pak $J$ Agric Sci 40: 1-2.

Sahrawat, K. L., M. P. Jones, S. Diatta, and A. Adam, 2001. Response of upland rice to fertilizer phosphorus and its residual value in an ultisol. Communication in Soil Sci and Plant Analysis 32(15-16): 2457-2468. doi: 10.1081/CSS120000384

Singh, S. N. and Lallu. 2005. Influence of different levels of nitrogen on its uptake and productive efficiency of paddy varieties. Indian J Plant Physiol 10(1): 94-96.

Yosef Tabar, S. 2012. Effect of nitrogen and phosphorus fertilizer on growth and yield of rice (Oryza sativa L). Intl J Agron Plant Prod 3(12): 579-584.

Yosef Tabar, S. 2013. Effect of nitrogen and phosphorus fertilizer on spikelet structure and yield in rice (Oryza sativa L). Intl J Agri Crop Sci. 5 (11): 1204-1208.

Yosefi, K. H., M. Galavi, M. Ramrodi, S. R. Mousavi, 2011. Effect of bio-phosphate and chemical phosphorus fertilizer accompanied with micronutrient foliar application a growth, yield and yield component of maize (single cross704). Australian J Crop Sci 175-180. 